\title{
IMPACT OF TRANSCATHETER CLOSURE OF ATRIAL SEPTAL DEFECT ON RIGHT VENTRICULAR SIZE AND FUNCTION BY ECHOCARDIOGRAPHY
}

\author{
By
Hatem Al-Samman Mohammed ${ }^{1}$, Sameh Refat Hassan Allam ${ }^{1}$, Ahmed Meawad Al-Emam ${ }^{2}$, Mohammad Ismail Al-Deftar ${ }^{1}$ and Ashraf Abd- Almonem Sayed Ahmed ${ }^{3}$
Departments of ${ }^{1}$ Cardiology, ${ }^{3}$ Clinical Pathology, Faculty of Medicine, Al-Azhar University, Department of ${ }^{2}$ Cardiology, National Heart Institute \\ Corresponding author: Hatem Al-Samman Mohammed, \\ E-mail: $\underline{\text { dr_hatem_2004@yahoo.com }}$
}

\begin{abstract}
Background: Percutaneous atrial septal defect (ASD) device closure is a safe and effective means of reducing or eliminating interatrial shunting. The response of the right heart to device closure is incompletely understood.

Objective: To evaluate the effects of transcatheter closure of secundum ASD on right ventricle size and function, that is, both systolic and diastolic by transthoracic echocardiography (TTE) over a 6 month period.

Patients and Methods: One hundred patients had 100 device implantations. The patients were assessed with echocardiography before, at 1 and 6 months after procedure, at Bab Al-Shaareya Hospital of Al-Azhar University, and National Heart Institute, Egypt during the period from April 2017 to October 2019.

Results: Mean age was (12.62 \pm 11.39 years). Maximum ASD size indexed to body surface area (BSA) was $($ Mean $\pm \mathrm{SD}=17.88 \pm 5.08 \mathrm{~mm} / \mathrm{m} 2)$. The device size ranged from $11 \mathrm{~mm}$ to $31 \mathrm{~mm}$. There was significant decrease from baseline to 1 month and baseline to 6 months after closure In the following indexed parameters: RV basal, mid, longitudinal diameters I, RV end diastolic area I, RV/LV end diastolic dimension ratio, RA end systolic area I and Estimated systolic pulmonary artery pressure (ESPAP), Also parameters of RV systolic functions such as; tricuspid annular plane systolic excursion (TAPSE), tricuspid annular systolic velocity (TASV or S/) and fractional area change (FAC\%), and diastolic dysfunction parameters; E/A and $\mathrm{E} / \mathrm{e} /,(\mathrm{p}=0.001)$. while RV index of myocardial performance (RIMP) showed significant reduction at one month $(0.39 \pm 0.03$ vs. $0.35 \pm 0.02 ; \mathrm{p}=0.001)$ and non-significant change at six months in comparison to baseline $(\mathrm{p}=0.083)$.
\end{abstract}

Conclusion: RV volumes decreased significantly in the first month after ASD device closure and continued up to 6 months. There was no change in global right ventricular systolic function but a high basal RV systolic function decreased after closure. Some patients had impaired diastolic function before closure of defect, which reversed to normal within 6 months after closure.

Keywords: Atrial septal defect, Right atrium, Right ventricle.

\section{INTRODUCTION}

The prevalence of congenital heart disease and ASDs has increased over the past 50 years. In the 1930 s congenital heart disease was diagnosed in less than 1 per 1000 live births. In recent years, 
congenital heart disease is diagnosed in 9 per 1000 live births. Atrial septal defects were identified between 1945 and 1949 in less than 0.5 live births per 1000. More recent epidemiologic data suggest that ASDs occur in 1.6 per 1000 live births (Chelu et al., 2019). The noted increase in prevalence is probably not due to an increase in disease as much as improvements in imaging modalities and training of practitioners. Several factors have been associated with the increased prevalence of congenital heart disease including advanced maternal age. Interestingly, economic and geographical differences have been noted in the diagnosis. Congenital heart disease is diagnosed more commonly in patients in developed countries who have higher incomes (van der Linde et al., 2011).

There are five types of atrial septal defects ranging from most frequent to least: patent foramen ovale, ostium secundum defect, ostium primum defect, sinus venosus defect, and coronary sinus defect (Celermajer, 2018). Small atrial septal defects usually spontaneously close in childhood. Large defects that do not close spontaneously may require percutaneous or surgical intervention to prevent further complications such as stroke, dysrhythmias, and pulmonary hypertension. If an ASD requires closure, options include percutaneous and surgical intervention. Indications for treatment include stroke, a hemodynamically significant shunt greater than 1.5:1 and evidence of systemic oxygen desaturation. Percutaneous transcatheter closure poses less risk for the patient, but it is only useful for the closure of ostium secundum defects (Yang and Wu, 2018).
The aim of this study was to assess RV functions by 2-D echocardiography in patients with secundum ASD before transcatheter device closure and after 1 month and 6 months.

\section{PATIENTS AND METHODS}

This was a prospective study. The study included 100 seccundum ASD patients with percutaneous transcatheter closure by device occluders, and we examined the effects of device closure on right ventricle size and functions, that is, both systolic and diastolic by transthoracic echocardiography (TTE) over a 6-month period in Bab Al-shaareya hospital of AlAzhar University and National Heart Institute, Egypt. Period from April 2017 to October 2019.

All patients signed informed consents, and the study was approved by the local ethics committee. Key inclusion criteria were male or female patients 3 years old or more, fulfilling the diagnosis of secondum type ASD clinically and echocardiographically, were indicated for closure of an ASD percutaneously a) Left to right shunt with a Qp/Qs ratio of $\geq 1.5: 1$, b) Dilatation of right heart chambers, c) History of paradoxical embolism and Successful device closure. and key exclusion criteria were: Previous myocardial infarction or angiographically confirmed coronary artery disease, severe valvular disease, Chronic obstructive pulmonary disease, Patients with depressed right and/or left ventricular function and Cardiomyopathy, Patients with significant rhythm and conduction disturbances (atrial flutter, atrial fibrillation, atrioventricular [AV] block, and left bundle branch block) and Refused 
or missed follow up or inadequate quality echo image.

Every patient included in this study was subjected to full medical history and and physical examination with emphasis on the New York heart association (NYHA) class, signs of pulmonary hypertension (accentuated second heart sound) and signs of RV volume overload, a standard 12 leads ECG ,chest X-ray and routine laboratory investigations.

All patients underwent full echocardiographic study 12-24 hours before, 1 and 6 months after successful closure of ASD percutaneously. Echocardiographic evaluation was performed in left lateral decubitus position using with a Philips iE33 echocardiography Machine. Measurements taken according to the American Society of Echocardiography recommendations (Lang et al., 2015).

To differentiate normal RV structure and function from abnormal and to assess RV size, volume, and contractility, a complete set of standardized views were obtained. These include left parasternal long-axis (PLAX), parasternal short-axis (PSAX), right ventricle- focused apical 4chamber (A4C), and subcostal views. To decrease the respiratory variations, all measurements were acquired at the end of expiration in three consecutive cycles and the average value was taken. RV basal, mid and longitudinal diameter were estimated at end-diastole from apical 4chamber view by obtaining the maximum measurement. Right ventricle/left ventricle (RV/LV) diameter ratio was obtained accordingly. In the same apical 4-chamber view at end-systole, right atrial area (RAA) was estimated. RVEDA and
RVESA were obtained by tracing the RV endocardium both in diastole (RVEDA) and systole (RVESA) from the annulus, along the free wall to the apex, and then back to the annulus, along the interventricular septum.

All measured dimensions were indexed to body surface area (BSA). Also in apical 4 chamber view by measuring tricuspid regurgitation (TR) velocity, estimated systolic pulmonary artery pressure (ESPAP) was calculated by modified Bernoulli equation (ESPAP) $=4(\mathrm{TR}$ velocity) $2+$ RA pressure.

After the assessment of dimensions, RV systolic function and diastolic function were assessed. Tricuspid annular plane systolic excursion (TAPSE) was measured from the tricuspid lateral annulus by M-mode. The percentage RV Fractional area change (FAC\%) was calculated by the formula: (RVenddiastolic area - RV end-systolic area)/ RVend-diastolic area X 100. By measuring tricuspid valve closure to opening time (TCOT) and RV ejection time (RV ET) from pulsed-wave Doppler in RVOT and RV inflow, RV index of myocardial performance (RIMP) or Tei index was estimated by the formula "RIMP = (TCOT-ET)/ET.". From the pulsed-wave (PW) Doppler tissue image, systolic annulus velocity (TASV or $\mathrm{S} /$ ) and early diastolic tricuspid annulus velocity (e/) were also measured. Early diastolic tricuspid flow velocity $(\mathrm{E})$, late diastolic tricuspid flow velocity (A), and deceleration time (DT) were estimated by pulsed-wave Doppler placed at the tips of tricuspid valve.

Statistical analysis: All data were collected, tabulated and statistically 
analyzed using the PC computer. The data were entered to the Statistical Package for the Social Science Program (SPSS) version 23.The quantitative data were presented as mean and SD and range for the parametric data and as median and interquartile ranges (IQR) for the nonparametric data while the qualitative data was presented as a number and percentage. Paired sample t-test was used to compare between two paired groups with quantitative data, unpaired t-test was used to compare means of 2 different groups, while Fredman test and Mann Whitney $U$ test were used for nonparametric test. A P-value $<0.05$ was considered statistically significant.

\section{RESULTS}

The total number of patients included in the study were 100 patients; sixty $(60 \%)$ were females and forty $(40 \%)$ were males, with age ranging from 3 to 50 years with mean $12.62 \pm 11.39$ years, The body surface area (BSA) of our patients ranged from 0.6-2.1 $\mathrm{m} 2$ with mean
$1.11 \pm 0.41 \mathrm{~m} 2$, The weight of our patients ranged from 15 to $90 \mathrm{~kg}$ with mean $35.24 \pm 18.99 \mathrm{~kg}$, The height ranged from $0.9 \mathrm{~m}$ to $1.8 \mathrm{~m}$ with mean $1.29 \pm 0.27 \mathrm{~m}$, The Body mass index ranged from 15.6 $\mathrm{kg} / \mathrm{m} 2$ to $29.1 \mathrm{~kg} / \mathrm{m} 2$ with mean $19.37 \pm 2.81 \mathrm{~kg} / \mathrm{m} 2$ (Table 1).

Table (1): Age, weight, Height, BSA, BMI and Gender of the studied patients

\begin{tabular}{|l|c|}
\hline \multicolumn{1}{|c|}{ Characteristics } & Value \\
\hline $\begin{array}{l}\text { Age (yrs) } \\
\text { Range (Mean } \pm \text { SD) }\end{array}$ & $3-50(12.62 \pm 11.39)$ \\
\hline $\begin{array}{l}\text { Weight(Kg) } \\
\text { Range (Mean } \pm \text { SD) }\end{array}$ & $15-90(35.24 \pm 18.99)$ \\
\hline $\begin{array}{l}\text { Height(m) } \\
\text { Range (Mean } \pm \text { SD) }\end{array}$ & $0.9-1.8(1.29 \pm 0.27)$ \\
\hline $\begin{array}{l}\text { BSA(m }\left(\mathbf{m}^{2}\right) \\
\text { Range }(M e a n \pm S D)\end{array}$ & $0.6-2.1(1.11 \pm 0.41)$ \\
\hline $\begin{array}{l}\left.\text { BMI(kg/m }{ }^{2}\right) \\
\text { Range (Mean } \pm \text { SD) }\end{array}$ & $15.6-29.1(19.37 \pm 2.81)$ \\
\hline $\begin{array}{l}\text { Gender } \\
\text { Male no. }(\%): \text { Female no. }(\%)\end{array}$ & $40(40 \%): 60(60 \%)$ \\
\hline
\end{tabular}

$\mathrm{BSA}=$ body surface area; $\mathrm{BMI}=$ body mass index

All patients had normal sinus rhythm, no hypertension and no diabetes mellitus in our studied patients. All the hundred patients had 1-month and 6-month followup. No residual interatrial shunt occurred in patients at 1 month and at 6 month during follow-up. The ASD maximum diameters were measured using TTE and TEE in studied patients, maximum diameters ranged from $11 \mathrm{~mm}$ to $31 \mathrm{~mm}$ with mean $18.43 \pm 4.50 \mathrm{~mm}$, and was indexed to body surface area and showed range from $9.7 \mathrm{~mm} / \mathrm{m} 2$ to $38.1 \mathrm{~mm} / \mathrm{m} 2$ with mean $17.88 \pm 5.08 \mathrm{~mm} / \mathrm{m} 2$. During procedure $\mathrm{QP} / \mathrm{QS}$ calculated, it ranged from1.7 to 3.2 with mean $2.30 \pm 0.38$, Device sizes used for closure ranged from $12 \mathrm{~mm}$ to $34 \mathrm{~mm}$ with mean $20.77 \pm 4.68$ mm (Table 2). 
Table (2): ASD Diameter, Qp/Qs and Device Size

\begin{tabular}{|l|c|c|c|}
\hline Variables & Minimum & Maximum & Mean \\
\hline ASD MAX D & 11 & 31 & $18.43 \pm 4.50$ \\
\hline ASD MAX Indexed & 9.7 & 38.1 & $17.88 \pm 5.08$ \\
\hline QP/QS & 1.7 & 3.2 & $2.30 \pm 0.38$ \\
\hline Device Size & 12 & 34 & $20.77 \pm 4.68$ \\
\hline
\end{tabular}

ASD Max D= atrial septal defect maximum diameter; QP/QS-pulmonary flow/systemic flow.

There was a significant decrease from baseline to 1 month $(\mathrm{p}=0.001)$ and baseline to 6 months $(\mathrm{p}=0.001)$ after device closure in the following parameters: RVBDI $(\mathrm{cm} / \mathrm{m} 2)$ $(3.77 \pm 0.94 \mathrm{vs} .3 .24 \pm 0.82 \quad$ vs. $3.04 \pm 0.74)$, RVMDI $(\mathrm{cm} / \mathrm{m} 2)(2.18 \pm 0.67$ vs. $1.79 \pm$ 0.51 vs. $1.77 \pm 0.50)$. RVLD I $(\mathrm{cm} / \mathrm{m} 2)$ $(5.62 \pm 1.61$ vs. $5.22 \pm 1.49$ vs. $4.86 \pm 1.40)$. RVEDA I $\quad(\mathrm{cm} 2 / \mathrm{m} 2) \quad(18.5 \pm 3.18$ vs. $16.98 \pm 3.06$ vs. $16.98 \pm 3.07)$. RV/LV EDD ratio $(1.1 \pm 0.08$ vs. $0.89 \pm 0.05$ vs. $0.81 \pm 0.034)$, and RAA I $(\mathrm{cm} 2 / \mathrm{m} 2)$ $(13.33 \pm 1.46$ vs. $10.44 \pm 0.84$ vs. 9.44 \pm 0.85$)$, ESPAP $(\mathrm{mmHg}) \quad(43.3 \pm 5.2$ vs.27.46 \pm 4.2 vs.19.53 \pm 3.4$)$, Also RV systolic functions parameters; TAPSE (cm) $\quad(2.86 \pm 0.19$ vs. $2.24 \pm 0.18$ vs. $2.46 \pm 0.15), \quad($ TASV or $\mathrm{S} /) \quad(\mathrm{cm} / \mathrm{sec})$ $(16.24 \pm 0.85$ vs. $13.1 \pm 0.42$ vs. $13.52 \pm 0.43)$ and FAC\% $(43.8 \pm 2.6$ vs.38.76 \pm 2.46 vs. $38.41 \pm 2.57$ ), and diastolic dysfunction parameters; E/A (1.7 \pm 0.14 vs. $1.36 \pm 0.09$ vs. $1.48 \pm 0.1)$ and $E / e /(6.9 \pm 0.78$ vs. $5.9 \pm$ 0.19 vs. $4.99 \pm 0.62)$. While RIMP and DT showed significant reduction at one month; RIMP $(0.39 \pm 0.03$ vs. $0.35 \pm 0.02$; $\mathrm{p}=0.001)$, DT (msec) $\quad(167.7 \pm 8.2$ vs. $167.3 \pm 7.6 ; \mathrm{p}=0.001)$ and non-significant change at six months in comparison to baseline (Table 3). 
HATEM A. MOHAMMED et al.,

Table (3): Comparison between RV echocardiographic parameters before and one month and sex months after device closure

\begin{tabular}{|c|c|c|c|c|c|}
\hline $\begin{array}{ll}\text { Variables } & \text { Time } \\
\end{array}$ & $\begin{array}{c}\text { Baseline } \\
(0)\end{array}$ & $\begin{array}{l}\text { 1-month } \\
\text { follow up }\end{array}$ & $\begin{array}{l}\text { 6-month } \\
\text { follow up }\end{array}$ & $\begin{array}{c}\text { p-value } \\
(0-1)\end{array}$ & $\begin{array}{c}\text { p-value } \\
(0-6)\end{array}$ \\
\hline RVBD I $\left(\mathbf{c m} / \mathbf{m}^{2}\right)$ & $3.77 \pm 0.94$ & $3.24 \pm 0,82$ & $3.04 \pm 0.74$ & 0.001 & 0.001 \\
\hline RVMD I (cm/m²) & $2.18 \pm 0.67$ & $1.79 \pm 0.51$ & $1.77 \pm 0.50$ & 0.001 & 0.001 \\
\hline RVLDI $\left(\mathrm{cm} / \mathrm{m}^{2}\right)$ & $5.62 \pm 1.61$ & $5.22 \pm 1.49$ & $4.86 \pm 1.40$ & 0.001 & 0.001 \\
\hline $\begin{array}{c}\text { RV/LV EDD } \\
\text { ratio }\end{array}$ & $1.1 \pm 0.08$ & $0.89 \pm 0.05$ & $0.81 \pm 0.034$ & 0.001 & 0.001 \\
\hline RAAI $\left(\mathbf{c m}^{2} / \mathbf{m}^{2}\right)$ & $13.33 \pm 1.46$ & $10.44 \pm 0.84$ & $9.44 \pm 0.85$ & 0.001 & 0.001 \\
\hline ESPAP (mmHg) & $43.3 \pm 5.2$ & $27.46 \pm 4.2$ & $19.53 \pm 3.4$ & 0.001 & 0.001 \\
\hline $\begin{array}{c}\text { RVEDA } \\
\mathbf{I}\left(\mathbf{c m}^{2} / \mathbf{m}^{2}\right)\end{array}$ & $18.5 \pm 3.18$ & $16.98 \pm 3.06$ & $16.98 \pm 3.07$ & 0.001 & 0.001 \\
\hline $\begin{array}{c}\text { RVESA } \\
\text { I }\left(\mathrm{cm}^{2} / \mathbf{m}^{2}\right)\end{array}$ & $10.39 \pm 1.78$ & $10.38 \pm 1.78$ & $10.44 \pm 1.84$ & 0.320 & 0.024 \\
\hline FAC $(\%)$ & $43.8 \pm 2.6$ & $38.76 \pm 2.46$ & $38.41 \pm 2.57$ & 0.001 & 0.001 \\
\hline TAPSE $(\mathrm{cm})$ & $2.86 \pm 0.19$ & $2.24 \pm 0.18$ & $2.46 \pm 0.15$ & 0.001 & 0.001 \\
\hline $\begin{array}{c}\text { TASV } \\
\left(\mathbf{S}^{\prime}\right)(\mathrm{cm} / \mathrm{sec}) \\
\end{array}$ & $16.24 \pm 0.85$ & $13.1 \pm 0.42$ & $13.52 \pm 0.43$ & 0.001 & 0.001 \\
\hline RIMP & $0.39 \pm 0.03$ & $0.35 \pm 0.02$ & $0.39 \pm 0.03$ & 0.001 & 0.083 \\
\hline $\mathbf{E} / \mathbf{A}$ & $1.7 \pm 0.14$ & $1.36 \pm 0.09$ & $1.48 \pm 0.1$ & 0.001 & 0.001 \\
\hline E/e' & $6.9 \pm 0.78$ & $5.9 \pm 0.19$ & $4.99 \pm 0.62$ & 0.001 & 0.001 \\
\hline DT (msec) & $167.7 \pm 8.2$ & $167.3 \pm 7.6$ & $167.6 \pm 8.32$ & 0.001 & 0.748 \\
\hline
\end{tabular}

$\mathrm{RV}=$ right ventricle; $\mathrm{BD} \mathrm{I}=$ basal diameter indexed; $\mathrm{MD} \mathrm{I}=$ mid diameter indexed; $\mathrm{LD} \mathrm{I}=$ longitudinal diameter indexed; $\mathrm{LV}=$ left ventricle $\mathrm{EDD}$ ratio= end-diastolic diameter ratio; RAA $\mathrm{I}=$ right atrium area indexed; ESPAP=estimated systolic pulmonary artery pressure; RVEDA I=RV end diastolic area indexed ;RVESA I=RV end systolic area indexed; FAC = fractional area change; TAPSE= tricuspid annular plane systolic excursion; TASV $\left(\mathrm{S}^{\prime}\right)=$ tricuspid annular systolic velocity; RIMP= right ventricle index of myocardial performance; $\mathrm{E} / \mathrm{A}=$ early diastolic tricuspid valve inflow/late diastolic tricuspid valve inflow; $\mathrm{e}^{\prime}=$ early diastolic tricuspid annular velocity; DT = deceleration time.

Patients were divided into group 1 (younger) age $\leq 5$ years 31 patients $(15$ females and 16 males) and group 2 (older) age $>5$ years 69 patients (45 females and 24 males) and we studied each group before, one month and six months after ASD closure.
Before ASD closure, group 1 showed statistically significant lower Age, Weight, Height, BSA, BMI, EPAP, E/e', DT, Devise size. And showed statistically significant greater ASD max I, RVB I, RVM I, RVL I, RVEDA I, RVESA I, TAPSE in comparison to group 2 (Table 4). 
Table (4): Comparison between group 1 and group 2 patients Before ASD closure

\begin{tabular}{|c|c|c|c|}
\hline $\begin{array}{ll}\text { Variables } & \text { Groups } \\
\end{array}$ & $\begin{array}{c}\text { Group } 1 \text { (age } \leq 5 \text { ys) } \\
(n=31)\end{array}$ & $\begin{array}{c}\text { Group } 2 \text { (age }>5 \text { ys) } \\
(n=69)\end{array}$ & P-value \\
\hline Age (years) & $3.74 \pm 0.77$ & $16.61 \pm 11.68$ & $<0.001$ \\
\hline Weight(kg) & $16.82 \pm 1.80$ & $43.51 \pm 17.31$ & $<0.001$ \\
\hline Height(m) & $0.97 \pm 0.04$ & $1.43 \pm 0.19$ & $<0.001$ \\
\hline $\operatorname{BSA}\left(\mathbf{m}^{2}\right)$ & $0.67 \pm 0.05$ & $1.31 \pm 0.34$ & $<0.001$ \\
\hline BMI $\left(\mathrm{kg} / \mathrm{m}^{2}\right)$ & $17.67 \pm 0.78$ & $20.13 \pm 3.06$ & $<0.001$ \\
\hline $\operatorname{ASD} \max I\left(\mathbf{m m} / \mathbf{m}^{2}\right)$ & $22.97 \pm 5.56$ & $15.59 \pm 2.62$ & $<0.001$ \\
\hline RVBD I $\left(\mathbf{c m} / \mathbf{m}^{2}\right)$ & $5.01 \pm 0.32$ & $3.22 \pm 0.48$ & $<0.001$ \\
\hline RVMD I $\left(\mathrm{cm} / \mathrm{m}^{2}\right)$ & $3.04 \pm 0.31$ & $1.79 \pm 0.35$ & $<0.001$ \\
\hline RVLD I $\left(\mathbf{c m} / \mathbf{m}^{2}\right)$ & $7.70 \pm 0.35$ & $4.68 \pm 0.92$ & $<0.001$ \\
\hline RV/LV EDD ratio & $1.10 \pm 0.08$ & $1.11 \pm 0.08$ & 0.564 \\
\hline RAA I $\left(\mathbf{c m}^{2} / \mathbf{m}^{2}\right)$ & $13.09 \pm 0.30$ & $13.44 \pm 1.74$ & 0.279 \\
\hline ESPAP(Mmhg) & $40.64 \pm 5.74$ & $44.50 \pm 4-63$ & $<0.001$ \\
\hline RVEDA I $\left(\mathrm{cm}^{2} / \mathrm{m}^{2}\right)$ & $22.05 \pm 1.71$ & $16.91 \pm 2.26$ & $<0.001$ \\
\hline RVESA I $\left(\mathrm{cm}^{2} / \mathbf{m}^{2}\right)$ & $12.32 \pm 0.99$ & $9.52 \pm 1.31$ & $<0.001$ \\
\hline FAC $(\%)$ & $44.11 \pm 2.18$ & $43.67 \pm 2.77$ & 0.440 \\
\hline TAPSE $(\mathbf{c m})$ & $2.93 \pm 0.16$ & $2.83 \pm 0.21$ & 0.025 \\
\hline TASV $\left(\mathbf{S}^{\prime}\right)(\mathrm{cm} / \mathrm{sec})$ & $16.42 \pm 0.60$ & $16.15 \pm 0.94$ & 0.136 \\
\hline RIMP & $0.39 \pm 0.02$ & $0.39 \pm 0.03$ & 0.257 \\
\hline E/A & $1.74 \pm 0.15$ & $1.71 \pm 0.13$ & 0.312 \\
\hline$E / e^{\prime}$ & $6.68 \pm 0.90$ & $7.04 \pm 0.66$ & 0.027 \\
\hline DT (msec) & $164.23 \pm 10.04$ & $169.30 \pm 6.82$ & 0.004 \\
\hline QP/QS & $2.25 \pm 0.37$ & $2.32 \pm 0.38$ & 0.424 \\
\hline Devise size(mm) & $17.71 \pm 3.55$ & $22.14 \pm 4.49$ & $<0.001$ \\
\hline
\end{tabular}

BSA= body surface area; BMI=body mass index; ASD Max I = atrial septal defect maximum indexed diameter; $\mathrm{RV}=$ right ventricle; $\mathrm{BD} \mathrm{I}=$ basal diameter indexed; $\mathrm{MD} \mathrm{I}=$ mid diameter indexed; $\mathrm{LD} \mathrm{I}=$ longitudinal diameter indexed; $\mathrm{LV}=$ left ventricle; $\mathrm{EDD}$ ratio= end-diastolic diameter ratio; RAA I= right atrium area indexed; ESPAP=estimated systolic pulmonary artery pressure; RVEDA I=RV end diastolic area indexed ;RVESA I=RV end systolic area indexed; $\mathrm{FAC}=$ fractional area change; TAPSE $=$ tricuspid annular plane systolic excursion; TASV $\left(\mathrm{S}^{\prime}\right)=$ tricuspid annular systolic velocity; RIMP= right ventricle index of myocardial performance; $\mathrm{E} / \mathrm{A}=$ early diastolic tricuspid valve inflow/late diastolic tricuspid valve inflow; $\mathrm{e}^{\prime}=$ early diastolic tricuspid annular velocity; DT= deceleration time; QP/QS=pulmonary flow/systemic flow.

After ASD closure, group 1 showed statistically significant more reduction in comparison to group 2 in the following parameters: indexed RVB diameter, indexed and percentage change of RVMI diameter, indexed RVL diameter and indexed RVEDA at one month and six months post-device closure. While group 2 showed statistically significant more reduction in comparison to group one in RV/LV EDD Ratio and TAPSE, also their percentage changes one month postdevice closure. And also group 2 showed statistically significant more reduction in indexed RAAI and ESPAP and their percentage changes at one month and six months post-device closure (Table 5). 
Table (5): Comparison between changes and percentage of changes occurred in right heart dimensions and functions in both groups at 1 month and 6 months after ASD closure

\begin{tabular}{|c|c|c|c|c|c|c|}
\hline \multirow{2}{*}{ Variables } & \multicolumn{3}{|c|}{ Baseline to one month (0-1) } & \multicolumn{3}{|c|}{ Baseline to six months (0-6) } \\
\hline & G1 $(n=31)$ & G2 $(n=69)$ & p-value & G1 $(n=31)$ & G2 $(n=69)$ & $\begin{array}{c}\text { p- } \\
\text { value }\end{array}$ \\
\hline $\operatorname{RVBD}$ I $\left(\mathbf{c m} / \mathbf{m}^{2}\right)$ & $0.68 \pm 0.23$ & $0.47 \pm 0.14$ & $<0.001$ & $1.01 \pm 0.25$ & $0.61 \pm 0.16$ & $<0.001$ \\
\hline Percent. & $0.14 \pm 0.04$ & $0.14 \pm 0.04$ & 0.306 & $0.25 \pm 0.06$ & $0.24 \pm 0.06$ & 0.287 \\
\hline RVMD I $\left(\mathbf{c m} / \mathrm{m}^{2}\right)$ & $0.60 \pm 0.24$ & $0.29 \pm 0.12$ & $<0.001$ & $0.62 \pm 0.26$ & $0.31 \pm 0.12$ & $<0.001$ \\
\hline Percent. & $0.18 \pm 0.03$ & $0.16 \pm 0.05$ & 0.022 & $0.24 \pm 0.05$ & $0.21 \pm 0.07$ & 0.034 \\
\hline $\operatorname{RVLD~I~}\left(\mathrm{cm} / \mathrm{m}^{2}\right)$ & $0.55 \pm 0.15$ & $0.33 \pm 0.07$ & $<0.001$ & $1.01 \pm 0.16$ & $0.64 \pm 0.49$ & $<0.001$ \\
\hline Percent. & $0.07 \pm 0.02$ & $0.07 \pm 0.01$ & 0.796 & $0.15 \pm 0.02$ & $0.17 \pm 0.17$ & 0.639 \\
\hline $\begin{array}{c}\text { RV/LV EDD } \\
\text { ratio }\end{array}$ & $0.19 \pm 0.06$ & $0.22 \pm 0.09$ & 0.035 & $0.29 \pm 0.08$ & $0.30 \pm 0.09$ & 0.642 \\
\hline Percent. & $0.17 \pm 0.04$ & $0.20 \pm 0.07$ & 0.032 & $0.36 \pm 0.10$ & $0.36 \pm 0.11$ & 0.680 \\
\hline RAAI $\left(\mathbf{c m}^{2} / \mathbf{m}^{2}\right)$ & $2.46 \pm 0.43$ & $3.09 \pm 1.11$ & 0.003 & $3.31 \pm 0.59$ & $4.15 \pm 1.22$ & $<0.001$ \\
\hline Percent. & $0.19 \pm 0.03$ & $0.23 \pm 0.06$ & $<0.001$ & $0.34 \pm 0.07$ & $0.45 \pm 0.13$ & $<0.001$ \\
\hline ESPAP(Mmhg) & $13.68 \pm 6.87$ & $16.83 \pm 5.88$ & 0.021 & $21.37 \pm 7.32$ & $24.86 \pm 5.40$ & 0.009 \\
\hline Percent. & $0.32 \pm 0.12$ & $0.37 \pm 0.11$ & 0.053 & $1.19 \pm 0.59$ & $1.34 \pm 0.48$ & 0.177 \\
\hline RVEDAI & & & & & & \\
\hline $\begin{array}{c}\left(\mathbf{c m}^{2} / \mathbf{m}^{2}\right) \\
\text { Percent. }\end{array}$ & $\begin{array}{l}1.96 \pm 0.84 \\
0.09 \pm 0.04\end{array}$ & $\begin{array}{l}1.32 \pm 0.80 \\
0.08 \pm 0.05\end{array}$ & $\begin{array}{c}<0.001 \\
0.223\end{array}$ & $\begin{array}{l}1.95 \pm 0.82 \\
0.10 \pm 0.05\end{array}$ & $\begin{array}{l}1.32 \pm 0.80 \\
0.09 \pm 0.05\end{array}$ & $\begin{array}{c}<0.001 \\
0.255\end{array}$ \\
\hline RVESAI & $0.02 \pm 0.12$ & $0.00 \pm 0.00$ & 0.136 & $-0.09 \pm 0.38$ & $-0.04 \pm 0.17$ & 0.404 \\
\hline$\left(\mathbf{c m}^{2} / \mathbf{m}^{2}\right)$ Percent. & $0.00 \pm 0.01$ & $0.00 \pm 0.00$ & 0.136 & $-0.01 \pm 0.03$ & $0.00 \pm 0.02$ & 0.741 \\
\hline FAC $(\%)$ & $5.49 \pm 2.33$ & $4.84 \pm 2.83$ & 0.266 & $5.97 \pm 2.45$ & $5.14 \pm 3.04$ & 0.185 \\
\hline Percent. & $0.12 \pm 0.05$ & $0.11 \pm 0.06$ & 0.236 & $0.16 \pm 0.07$ & $0.14 \pm 0.08$ & 0.203 \\
\hline TAPSE (cm) & $0.58 \pm 0.12$ & $0.64 \pm 0.11$ & 0.016 & $0.39 \pm 0.20$ & $0.40 \pm 0.16$ & 0.772 \\
\hline Percent. & $0.20 \pm 0.04$ & $0.23 \pm 0.03$ & $<0.001$ & $0.16 \pm 0.09$ & $0.17 \pm 0.06$ & 0.599 \\
\hline TASV(S') & & & & & & \\
\hline$(\mathrm{cm} / \mathrm{sec})$ & $3.30 \pm 0.57$ & $3.07 \pm 0.89$ & 0.204 & $2.75 \pm 0.74$ & $2.69 \pm 0.84$ & 0.732 \\
\hline Percent. & $0.20 \pm 0.03$ & $0.19 \pm 0.05$ & 0.180 & $0.20 \pm 0.06$ & $0.20 \pm 0.06$ & 0.853 \\
\hline RIMP & $0.04 \pm 0.03$ & $0.04 \pm 0.03$ & 0.408 & $0.00 \pm 0.00$ & $0.00 \pm 0.00$ & 0.243 \\
\hline Percent. & $0.09 \pm 0.06$ & $0.10 \pm 0.08$ & 0.403 & $0.00 \pm 0.00$ & $0.00 \pm 0.01$ & 0.243 \\
\hline $\mathbf{E} / \mathbf{A}$ & $0.36 \pm 0.15$ & $0.36 \pm 0.17$ & 0.977 & $0.25 \pm 0.16$ & $0.24 \pm 0.17$ & 0.676 \\
\hline Percent. & $0.20 \pm 0.07$ & $0.21 \pm 0.09$ & 0.861 & $0.17 \pm 0.011$ & $0.17 \pm 0.12$ & 0.807 \\
\hline$E / e^{\prime}$ & $0.86 \pm 0.76$ & $1.11 \pm 0.67$ & 0.103 & $1.85 \pm 0.85$ & $1.98 \pm 0.98$ & 0.527 \\
\hline Percent. & $0.12 \pm 0.10$ & $0.15 \pm 0.09$ & 0.109 & $0.39 \pm 0.18$ & $0.41 \pm 0.21$ & 0.632 \\
\hline DT (msec) & $0.48 \pm 1.06$ & $0.38 \pm 0.67$ & 0.541 & $0.45 \pm 5.07$ & $-0.06 \pm 1.62$ & 0.450 \\
\hline Percent. & $0.00 \pm 0.01$ & $0.00 \pm 0.00$ & 0.510 & $0.00 \pm 0.04$ & $-0.00 \pm 0.01$ & 0.372 \\
\hline
\end{tabular}

$\mathrm{RV}=$ right ventricle; $\mathrm{BD} \mathrm{I}=$ basal diameter indexed; $\mathrm{MD} \mathrm{I}=$ mid diameter indexed; LD I= longitudinal diameter indexed; $L V=$ left ventricle EDD ratio= end-diastolic diameter ratio; RAA I= right atrium area indexed; ESPAP=estimated systolic pulmonary artery pressure; RVEDA I=RV end diastolic area indexed; RVESA I=RV end systolic area indexed; FAC = fractional area change; TAPSE= tricuspid annular plane systolic excursion; TASV $\left(\mathrm{S}^{\prime}\right)=$ tricuspid annular systolic velocity; RIMP = right ventricle index of myocardial performance; $\mathrm{E} / \mathrm{A}=$ early diastolic tricuspid valve inflow/late diastolic tricuspid valve inflow; $\mathrm{e}^{\prime}=$ early diastolic tricuspid annular velocity; DT = deceleration time. 


\section{DISCUSSION}

Likely to our study, Kaya et al. (2010) performed Secundum-type ASD transcatheter closure in 117 patients (48 males and 69 females) aging from $15 \pm 12$ years (range, 2-65 years), and the mean follow-up period was $25.9+/-12.4$ months. They found that Transcatheter ASD closure leads to a significant improvement in clinical status and heart cavity dimensions in adults and children, as shown by intermediate-term follow-up evaluation.

In our study, we observed statistically significant decrease in multiple echocardiographic RV indexed dimensions from baseline to 1 month then to 6 months post device closure such as RVBD I, RVMD I, RVLD I, RVEDA I, $\mathrm{RV} / \mathrm{LV}$ EDD ratio, RAA I.

This agreed with Akula et al. (2016) who showed statistically significant decreases in RV BD; $16 \%$ decrease at one month after closure; and RV LD, that is $8 \%$ decrease at one month and further $6 \%$ decrease at six months from 1 month postASD device closure and RV/LV enddiastolic diameter ratio showed significant decrease; $22 \%$ decrease at one month post device closure, and further $10 \%$ decreased at six months from 1 month post-ASD device closure. Also RA end-systolic area showed significant decrease $28 \%$ at one month, and further $14 \%$ decrease at six months from 1 month post-ASD device closure. Also, Humenberger et al. (2011) stated that RV diameter decreased from 43 $\pm 7 \mathrm{~mm}$ at baseline to $34 \pm 6 \mathrm{~mm}$ after $3-6$ months and the decrease in RV size was observed in all age groups.

Global assessment of RV function includes the right ventricle index of myocardial performance (RIMP) and $\mathrm{FAC} \%$, while regional functional assessment includes M-mode-derived TAPSE, and the tissue Doppler-derived tricuspid annular velocity $\left(\mathrm{S}^{\prime}\right)$.

A simple, easily recordable, noninvasive Doppler right ventricle index of myocardial performance (RIMP) or Tei index as a sign of overall cardiac function. A RIMP value $\leq 0.43$ is regarded as normal RV function (Lang et al., 2015). In our study, RIMP was found to be normal at baseline and significant decrees was observed after closure at the first month then showed no significant change at six month in comparison with baseline. This disagreed with Akula et al. (2016) as RV MPI was found to be abnormal at baseline and no significant change was observed after closure at the first month.

In our study, we observed statistically significant decrease in $\mathrm{FAC} \%$ on the first post-interventional month and 6 months.

Significant decrease in TAPSE $(\mathrm{cm})$ occurred after closure of ASD on the first post-interventional month and at 6 months. Similar significant decrease had been reported by Vitarelli et al. (2011) at 6 months after closure.

TASV is a tissue Doppler-derived parameter which can be used to predict RV systolic function. A value of systolic annular velocity $<11.5 \mathrm{~cm} / \mathrm{sec}$ can predict a RV EF < 50\%, with $90 \%$ sensitivity and $85 \%$ specificity. In our study, as compared to previous values, we have shown significant decrease in TASV (S') $(\mathrm{cm} / \mathrm{sec})$ on the first post-interventional month and 6 months compared with baseline. Similarly, in their study, Zhang et al. (2010) have demonstrated decreases in tricuspid systolic annular velocity 3-5 
days after ASD closure as compared to values before closure.

A $\breve{g} a c ̧$ et al. (2012) evaluated RV function in early period following transcatheter closure of ASD, investigators observed there was significant decrease in TASV, and TAPSE which is concordant with our result, but no significant changes were observed in RV MPI which is disconcordant with our result as we observed significant reduction after one month of closure.

In our study, we have demonstrated that some indices of RV function such as FAC\%, TAPSE and TASV (S') showed significant decrease after closure of ASD in the first month then remaining stable when followed at six months. Similar changes had been reported by Akula et al. (2016), as they found after 1 month post device closure there were statistically significant decrease in FAC, and 8\% decrease from baseline, TAPSE, 9\% decrease from baseline and TASV, 18\% decrease from baseline. Six months after device closure, they observed no significant differences in FAC, TAPSE and TASV in comparison with 1 month post-device closure.

The results of the study of Akula et al. (2016) and our study are compatible with well-known physiological distinctions of right ventricle; The RV will accommodate lower volume after closure of ASD, which leads to pump sufficient stroke volume at decreased functional state. The increased preload as shown by EDV in right ventricle had high basal RV systolic function following Starling's law of the heart (Opie, 2010). The load dependent parameters such as FAC, TAPSE and $\mathrm{S}^{\prime}$ had significantly decreased post-ASD device closure creating a doubt of $\mathrm{RV}$ systolic dysfunction, but are normal according to the ASE guidelines (Lang et al., 2015), then remaining stable and within normal thereafter at six months when compared to one month values, This may explain the observation of high normal baseline RV systolic function in ASD patients which normalizes after device closure.

In our study, ESPAP decreased at baseline on the first post-interventional month to 6 months $(\mathrm{p}=0.001)$. This is agreed with Humenberger et al. (2011) as PAP decreased at base line, while 3 months after ASD closure remaining stable thereafter. That decrease was observed in all age groups.

$\mathrm{E}$ and $\mathrm{A}$ are load dependent and tissue doppler $\mathrm{e}^{\prime}$ is less load dependent and hence $\mathrm{E} / \mathrm{e}^{\prime}$ had gained the importance for measurement of diastolic dys-function. E/e' > 6 denotes diastolic dysfunction according to ASE guidelines (Lang et al., 2015).

In our study, we followed the diastolic functions for all patients, and found statistically significant decrease in parameters of RV diastolic function post device closure as compared with baseline, E/e' decreased at baseline to on the first post-interventional month, and at 6 months. DT (msec) decreased from baseline to the first post-interventional month and showed no significant change at 6 months in comparison to baseline.

In agreement to our study, Akula et al. (2016) found that after 1 month postdevice closure, there were statistically significant decrease in E/A, 16\% decrease from baseline and E/e' 18\% decrease from baseline. Six months after device closure, 
there were significant decrease in E/e' and $20 \%$ decrease from 1 month post-device closure. Six months after device closure, there are mild increase in E/A and 6\% increase from 1 month post-device closure. There was no significant difference in DT from baseline to 1 month and 6 months post-device closure. There were 49 patients with $\mathrm{E} / \mathrm{e}^{\prime}>6$ before ASD device closure which reduced to 17 patients within 6 months after device closure.

By comparing our studied age groups, we found that the demographic characteristics of group ONE (younger group age $\leq 5$ years) showed significant less age, BSA and BMI $(\mathrm{p}=0.00)$. This younger aged group showed statistically significant higher ASD maxI, RVBDI, RVMDI, RVLDI, RVEDAI, RVESAI and TAPSE at base line before the procedure compared to group TWO (older group age $>5$ years).

In our study, younger age group had significant lower ESPAP, which was matched with multivariate analysis proved that the probability of pulmonary hypertension significantly increased with a factor of 1.041 for each extra year of age in secundum ASD (Cossio-Aranda et al., 2016).

Yong et al. (2010) observed factors associated with a reduction in PASP after transcatheter ASD closure were younger age and smaller size (ie, body surface area). This is agreed with our study as we observed significant more reduction in ESPAP (mmHg) in group 2 (older) at one month compared to group 1 which continued at 6 months. Humenberger et al. (2011) found that at any age, ASD closure is followed by symptomatic improvement and regression of PAP and RV size. However, the best outcome is achieved in patients with less functional impairment and less elevated PAP. Considering the continuous increase in symptoms, RV remodeling, and PAP with age, ASD closure must be recommended irrespective of symptoms early after diagnosis.

Dehghani and Boyle (2012) experienced reduction in PA pressure, RV size and an improvement in functional capacity after percutaneous device closure of ASD, and these improvements appear to be greater if the defect is closed earlier.

In a study by Saito et al. (2012) to assess the natural history of medium-sized atrial septal defect in pediatric cases elucidated that Qp/Qs, RVEDV/LVEDV tended to increase more in the before school age group. The increasing ASD shunt in childhood may be related expansion of RVEDV by increased RV compliance other than increasing ASD diameter with age. Closure of the ASD results in volume unloading of the right heart and an early reduction in cardiac size.

In our study, there was a significant marked reduction of RVBD I $(\mathrm{cm} / \mathrm{m} 2)$ in group one versus group two at one month post device closure, Also, the same was observed at 6 months, but the percentage of change was not significantly changed. RVMD I $(\mathrm{cm} / \mathrm{m} 2)$ showed significant marked reduction in group one vs group two at one month, also at 6 months, Also, percentage of change was significantly more decreased in group one in comparison to group two $18 \%$ vs $16 \%$ at one month, and $24 \%$ vs $21 \%$ at 6 months. RVLD I (cm/m2) showed significant 
marked reduction in younger group compared to older group at one month and at 6 months, but the percentage of change was not significantly changed.

In our study, there was a significant more reduction of $\mathrm{RV} / \mathrm{LV}$ EDD ratio in group two in comparison to group one. Also the percentage of $\mathrm{RV} / \mathrm{LV}$ EDD ratio decrease was $20 \%$ in group two vs $17 \%$ in group one at one month which was significant and no significant change between both groups at 6 months. RAAI $(\mathrm{cm} 2 / \mathrm{m} 2)$ showed significant marked reduction in group two compared to group one at one month and 6 months. Also, percentage of change showed significantly more reduction in group two in comparison to group one $23 \%$ vs $19 \%$ at one month, and $45 \%$ vs $34 \%$ at 6 months.

In group ONE, there was significant more reduction in RVEDAI $(\mathrm{cm} 2 / \mathrm{m} 2)$ from base line to one month in comparison to group two; $(1.96 \pm 0.84$ vs $1.32 \pm 0.80 ; \quad \mathrm{p}=0.00)$ and continued significantly at 6 months $(1.95 \pm 0.82 \mathrm{Vs}$ $1.32 \pm 0.80 ; \mathrm{p}=0.00$ ) but the percentage of change was not significant between both groups. On the other hand no significant change between both groups at 1month or 6 months was observed in RVESAI or FAC $\%$.

TAPSE is the standard parameter for evaluating right ventricular function quantitatively (Sun et al., 2016). In our study, TAPSE $(\mathrm{cm})$ showed significantly more reduction in group two(older) at one month compared to group one and the percentage of change as well $23 \%$ vs $20 \%$, but non-significant change at 6 months was observed, while no significant change between both groups at 1month or 6 months was observed in TASV (S') $(\mathrm{cm} / \mathrm{sec})$ and RIMP.

\section{CONCLUSION}

This study demonstrated a reduction in right heart chamber sizes and normalization of high normal baseline RV systolic function more in the first month post-ASD device closure and continues beyond 6 months post procedure. This study also demonstrated that indices of RV function like RIMP, FAC, TASV and TAPSE may deteriorate after percutaneous closure of ASD, but the observed deterioration do not reflect a true functional loss. Instead, it is the result of normalization of cardiac geometry after shunt disappearance. At any age, ASD closure is followed by symptomatic improvement and regression of PAP and RV size. However, the best outcome is achieved in patients with less functional and cardiac geometry impairment; Younger children $\leq 5$ years showed more and faster improvement in RV size and dimensions and less deterioration in TAPSE .

\section{REFERENCES}

1. Ăgaç MT, Akyüz AR, Acar Z, Akdemir R, Korkmaz L, Kırış A and Celik S. (2012): Evaluation of right ventricular function in early period following transcatheter closure of atrial septal defect. Echocardiography, 29(3):358-62.

2. Akula VS, Durgaprasad R, Velam V, Kasala L, Rodda $M$ and Erathi HV. (2016): Right ventricle before and after atrial septal defect device closure. Echocardiography, 33(9):1381.

3. Celermajer DS. (2018): Atrial septal defects: even simple congenital heart diseases can be complicated. Eur Heart J., 39(12):9991001 . 
4. Chelu RG, Horowitz M, Sucha D, Kardys I, Ingremeau D, Vasanawala $S$ and Hsiao A. (2019): Evaluation of atrial septal defects with 4D flow MRI-multilevel and interreader reproducibility for quantification of shunt severity. MAGMA, 32(2):269-279.

5. Cossio-Aranda J, Zamora KD, Nanda NC, Uzendu A, Keirns C, Verdejo-Paris $J$ and Espinola-Zavaleta N. (2016): Echocardiographic correlates of severe pulmonary hypertension in adult patients with ostium secundum atrial septal defect. Echocardiography, 33:1891-6.

6. Dehghani $H$ and Boyle AJ. (2012): Percutaneous device closure of secundum atrial septal defect in older adults. Am J Cardiovasc Dis., 2(2):133-42.

7. Humenberger M, Rosenhek R, Gabriel H, Rader F, Heger M, Klaar $U$ and Baumgartner H. (2011): Benefit of atrial septal defect closure in adults: impact of age. European Heart Journal, 32(5): 553-560.

8. Kaya MG, Baykan A, Dogan A, Inanc T, Gunebakmaz O, Dogdu $O$ and Narin N. (2010): Intermediate - term effects of transcatheter secundum atrial septal defect closure on cardiac remodeling in children and adults. Pediatrcardiol., (31):474-482.

9. Lang RM, Badano LP, Mor-Avi V, Afilalo J, Armstrong A, Ernande L and Voigt JU. (2015): Recommendations for cardiac chamber quantification by echocardiography in adults: an update from the American Society of Echocardiography and the European Association of Cardiovascular Imaging. J Am Soc Echocardiogr., 28(1):139.

10. Opie LH. (2010): Mechanisms of cardiac contraction and relaxation. In: Libby $\mathrm{P}$, Bonow RO, Mann DL, Zipes DP (eds): Heart Disease: A Textbook of Cardiovascular Medicine, 8th ed.Pbl. Philadelphia: WB Saunders, Pp. 509-539.

11. Saito T, Ohta $K$, Nakayama $Y$, Hashida Y, Maeda A, Maruhashi K and
Yachie A. (2012): Natural history of medium-sized atrial septal defect in pediatric cases. Journal of Cardiology, 60(3): 248-251.

12. Sun $X$, Zhang $H$, Aike B, Yang $S$, Yang $Z$, Dong $L$, Wang $F$ and Wang C. (2016): Tricuspid annular plane systolic excursion (TAPSE) can predict the outcome of isolated tricuspid valve surgery in patients with previous cardiac surgery. J Thorac Dis., 8(3):369-374.

13. van der Linde $D$, Konings EE, Slager MA, Witsenburg M, Helbing WA, Takkenberg JJ and Roos-Hesselink JW. (2011): Birth prevalence of congenital heart disease worldwide: a systematic review and metaanalysis. J Am Coll Cardiol., 58(21):2241-7.

14. Vitarelli A, Di Roma A, Mancone M, Battaglia D, Caranci F, Capotosto $L$ and Rosanio S. (2011): Assessment of right ventricular function by three-dimensional and myocardial imaging echocardiography after percutaneous atrial septal defect closure in adults. Circulation, 120:S553.

15. Yang MC and Wu JR. (2018): Recent review of transcatheter closure of atrial septal defect. Kaohsiung J Med Sci., 34(7):363-369.

16. Yong $G$, Khairy $P$, De Guise $P$, Dore A, Marcotte F, Mercier LA and Ibrahim R. (2010): Pulmonary arterial hypertension in patients with transcatheter closure of secundum atrial septal defects: a longitudinal study. Circ Cardiovasc Interv., 2(5):455-6.

17. Zhang LH, Xu WH, Wang Y, Liu AQ, Lin CY, Li ZA and Zhang C. (2010): Evaluation of right ventricular function of patients with intraoperative device closure of atrial septal defect by ultrasonic Doppler tissue imaging. Zhonghua Yi Xue Za Zhi., 89(23):1627- 1629. 
تأثير غلق ثقب الحاجز الأذّنيني عن طريق القسطرة على حجم

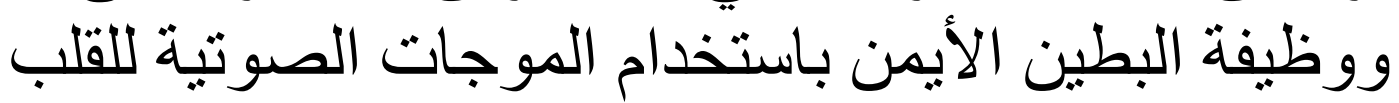
حاتم السمان محمد السمان1, سامح رفعت حسن علام¹, أحمد معوض الامام², محمد اسماعيل عبد الخالق الدفتار1, أثرف عبد المنعم سيد أحمد3

قسمي 1أمراض القلب والأوعية الدموية و 3الباثولوجيا الإلينيكية، كلية الطب، جامعة الأزهر قسم 2أمراض القلب والأوعية الدموية, معهز القلب القومي

E-mail: dr_hatem_2004@yahoo.com

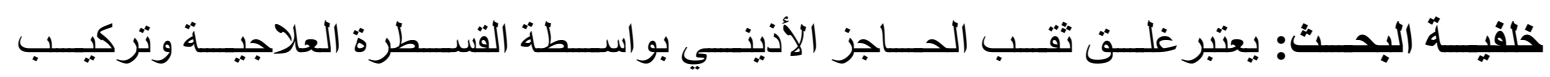

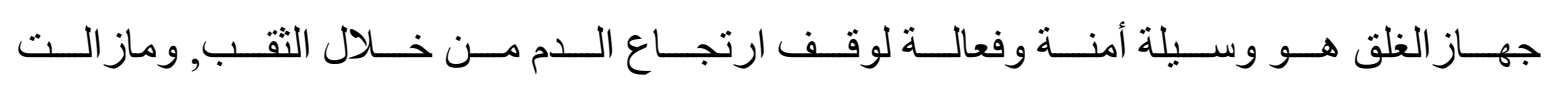

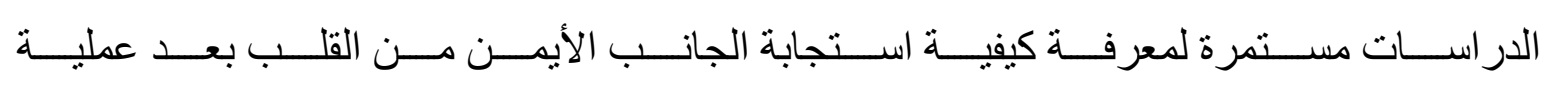
الغلق.

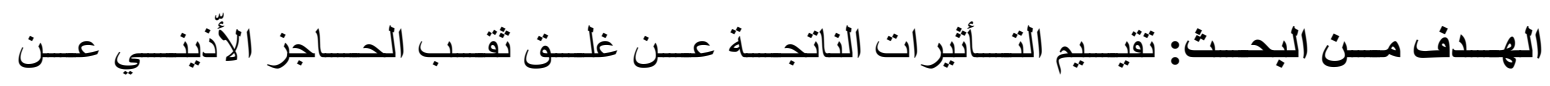

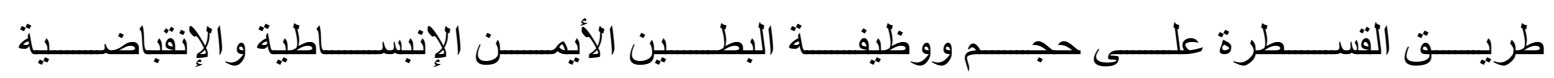
باستخدام الموجات الصوتية على القلب في فترة ستة أنثر.

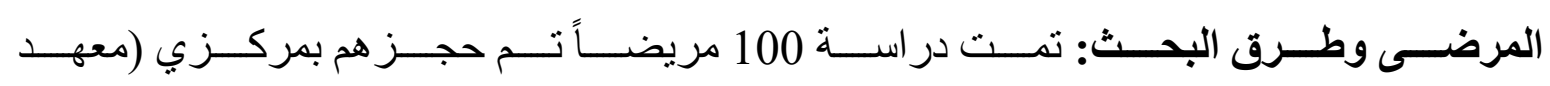

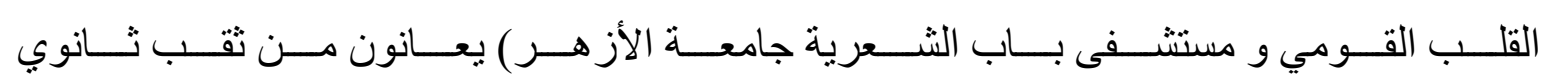

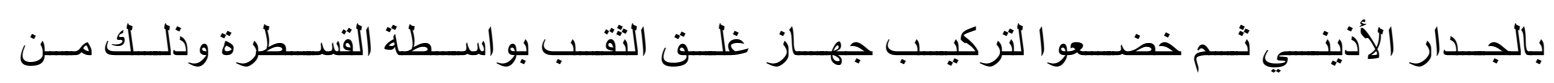

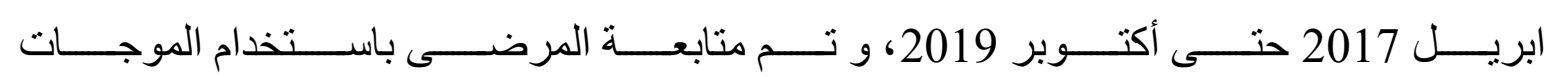
الصوتية على القلب قبل الغلق وبعد شهر وكذلك ستة أشهر بعد الغلق.

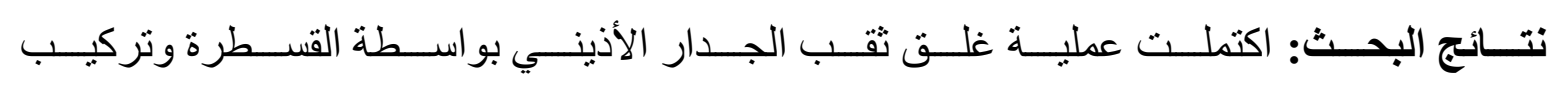

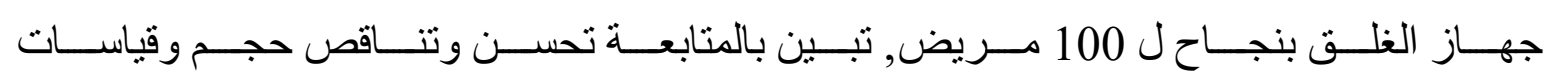

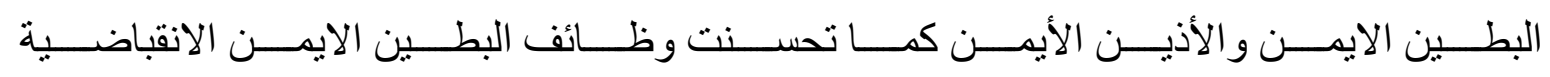

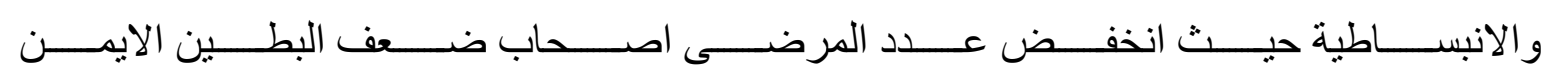

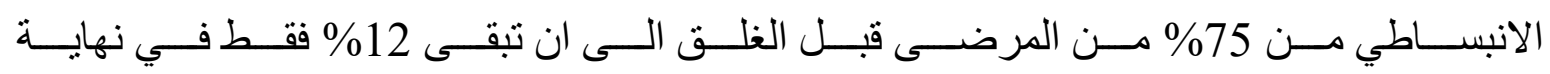

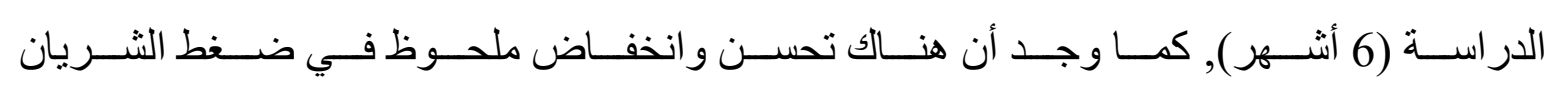




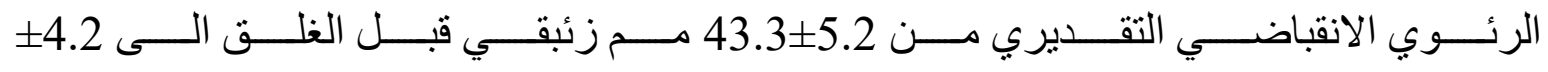

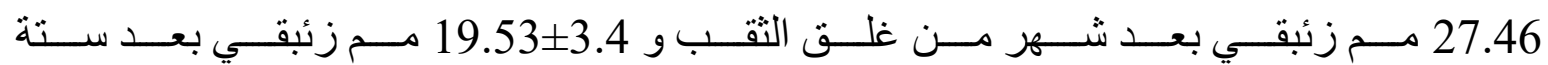

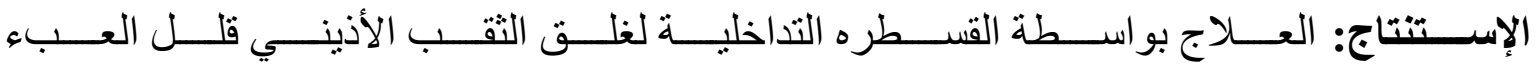

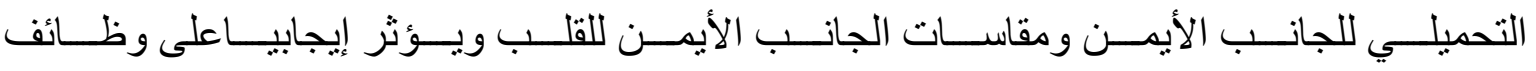
البطين الايمن وكذللك على إعادة هيكلة عضلة القلب فى الأطفال و الكبار. الكلمات الدالة: ثقب الحاجز الأذيني, الأذين الأيمن, البطين الأيمن. 